\title{
Exactly quantized dynamics of classical incommensurate sliders
}

\author{
Andrea Vanossi \\ CNR-INFM National Research Center S3 and Department of Physics, \\ University of Modena and Reggio Emilia, Via Campi 213/A, 41100 Modena, Italy \\ Nicola Manini and Giorgio Divitini \\ Dipartimento di Fisica and CNR-INFM, Università di Milano, Via Celoria 16, 20133 Milano, Italy and \\ International School for Advanced Studies (SISSA), Via Beirut 2-4, I-34014 Trieste, Italy \\ Giuseppe E. Santoro and Erio Tosatti \\ International School for Advanced Studies (SISSA), Via Beirut 2-4, I-34014 Trieste, Italy \\ INFM Democritos National Simulation Center and \\ International Centre for Theoretical Physics (ICTP), P.O.Box 586, I-34014 Trieste, Italy
}

(Dated: October 8, 2018)

\begin{abstract}
We report peculiar velocity quantization phenomena in the classical motion of an idealized 1D solid lubricant, consisting of a harmonic chain interposed between two periodic sliders. The ratio $v_{c m} / v_{\text {ext }}$ of the chain center-of-mass velocity to the externally imposed relative velocity of the sliders stays pinned to exact "plateau" values for wide ranges of parameters, such as sliders corrugation amplitudes, external velocity, chain stiffness and dissipation, and is strictly determined by the commensurability ratios alone. The phenomenon is explained by one slider rigidly dragging the kinks that the chain forms with the other slider. Possible consequences of these results for some real systems are discussed.
\end{abstract}

PACS numbers: 68.35.Af, 05.45.Yv, 62.25.+g, 62.20.Qp, 81.40.Pq, 46.55.+d

In this Letter we report surprising exact velocity quantization phenomena in a one-dimensional (1D) non-linear model inspired by the tribological problem of two sliding surfaces with a thin solid lubricant layer in between [1, 6]. The model layer consists of a chain of $N$ harmonically interacting particles interposed between two rigid generally (but not necessarily) incommensurate sinusoidal substrates (the two "sliding crystals", sketched in Fig. 1 1 ) externally driven at a constant relative velocity $v_{\text {ext }}$. The equation of motion of the $i$-th particle is:

$$
\begin{aligned}
m \ddot{x}_{i} & =-\frac{1}{2}\left[F_{+} \sin k_{+}\left(x_{i}-v_{+} t\right)+F_{-} \sin k_{-}\left(x_{i}-v_{-} t\right)\right] \\
& +K\left(x_{i+1}+x_{i-1}-2 x_{i}\right)-\gamma \sum_{ \pm}\left(\dot{x}_{i}-v_{ \pm}\right)
\end{aligned}
$$

where $m$ is the mass of the $N$ particles, $K$ is the chain spring constant, and $k_{ \pm}=(2 \pi) / a_{ \pm}$are the wave-vector periodicities of potentials representing the two sliders, moving at velocities $v_{ \pm}$. We set, in full generality, $v_{+}=$ 0 and $v_{e x t}=v_{-}-v_{+} \cdot \gamma$ is a phenomenological parameter substituting for various sources of dissipation, required to achieve a stationary state, but otherwise with no major role in the following. $F_{ \pm}$are the amplitudes of the forces due to the sinusoidal corrugation of the two sliders (we will commonly use $F_{-} / F_{+}=1$ but we checked that our results are more general). We take $a_{+}=1, m=1$, and $F_{+}=1$ as our basic units. The relevant length-ratios [2,3] are therefore $r_{ \pm}=a_{ \pm} / a_{0}$; we will take, without loss of generality, $r_{-}>r_{+}$, and confine our attention mostly to cases with $r_{+}>1$. The inter-particle equilibrium length $a_{0}$, not entering explicitly the equation of motion (1), appears only via the boundary conditions, which are taken to be periodic (PBC) [4], $x_{N+1}=x_{1}+N a_{0}$, to enforce a fixed density condition for the chain [5], with a coverage $r_{+}$of chain atoms on the denser substrate. Previous studies on a related model [6] achieved the sliding through the application of a constant driving to one of the two substrates, via an additional spring. That procedure obscures the surprising quantization phenomena which are instead uncovered when sliding occurs with a constant velocity $v_{\text {ext }}$.

Upon sliding the substrates, $v_{\text {ext }} \neq 0$, the lubricant chain slides too. However, it generally does so in a very unexpected manner: the time-averaged chain velocity $w=v_{c m} / v_{\text {ext }}$, is generally asymmetric, namely different from $1 / 2$. Even more surprisingly, $w$ is exactly quantized, for large parameter intervals, to plateau values that depend solely on the chosen commensurability ratios. The asymmetrical $w$-plateaus are generally very stable, and insensitive to many details of the model, due to their intrinsically topological nature; we show that they are the manifestation of a certain density of solitons in the lubricant which are set into motion by the external driving.

We now turn to illustrating these results. We integrated the equations of motion (10) starting from fully relaxed springs $\left(x_{i}=i a_{0}, \dot{x}_{i}=v_{e x t} / 2\right)$, by a standard fourth-order Runge-Kutta method. After an initial transient, the system reaches its dynamical stationary state, at least so long as $\gamma$ is not exactly zero. Figure 1r shows the resulting time-averaged center-of-mass $(\mathrm{CM})$ velocity $v_{c m}$ as a function of the chain stiffness $K$ for four repre- 


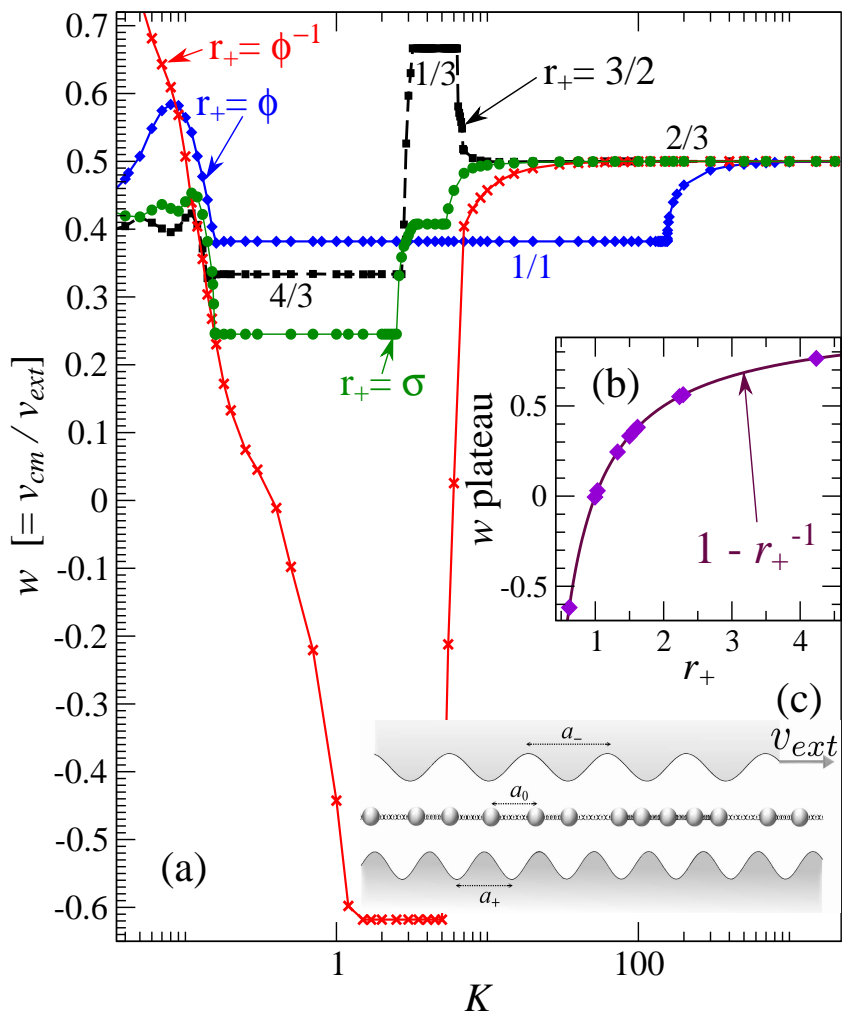

FIG. 1: (Color online) (a) Average drift velocity ratio $w=v_{c m} / v_{\text {ext }}$ of the chain as a function of its spring stiffness $K$ for different length ratios $\left(r_{+}, r_{-}\right)$: commensurate $(3 / 2,9 / 4)$, golden mean $(\mathrm{GM})\left(\phi, \phi^{2}\right)$, spiral mean (SM) $\left(\sigma, \sigma^{2}\right)\left(\sigma \sim 1.3247 \ldots\right.$ is the solution of $\left.\sigma^{3}=\sigma+1\right)$, and $\left(\phi^{-1}, \phi\right)$. The plateau labeling is explained in the text. Here $\gamma=0.1, v_{\text {ext }}=0.1$, and periodic boundary conditions [4] are used. The $\left(\phi, \phi^{2}\right) 1 / 1$ plateau value is $w=0.381966 \ldots$, identical to $1-\phi^{-1}$ to eight decimal places. (b) The main plateau speed $w$ as a function of $r_{+}$. (c) A sketch of the model.

sentative $\left(r_{+}, r_{-}\right)$values. We find that $w$ is generally a complicated function of $K$, with flat plateaus and regimes of continuous evolution, in a way which is qualitatively similar for different cases. The main surprise is that all plateaus show perfectly flat $w=v_{c m} / v_{\text {ext }}$ that are constant (quantized) to all figures of numerical accuracy, the precise value strikingly independent not only of $K$, but also of $\gamma, v_{e x t}$, and even of $F_{-} / F_{+}$. Open-boundary simulations show moreover that the $\mathrm{PBC}$ used are not crucial to the plateau quantization, which occurs even for a lubricant of finite size and not particularly large $N$, such as a hydrocarbon chain molecule would be.

To explore the origin of the universality of $w$, we analyze the dynamics for a large number of values of $\left(r_{+}, r_{-}\right)$, and observe that: (i) at least one velocity plateau as a function of $K$ occurs for any $\left(r_{+}, r_{-}\right)$; (ii) additional narrower secondary plateaus often arise for stiffer lubricant (larger $K$, see Fig. (1) ; (iii) the velocity ratio $w$ of the first plateau found for increasing $K$, satisfies $w=1-r_{+}^{-1}$ for a large range of $\left(r_{+}, r_{-}\right)$.

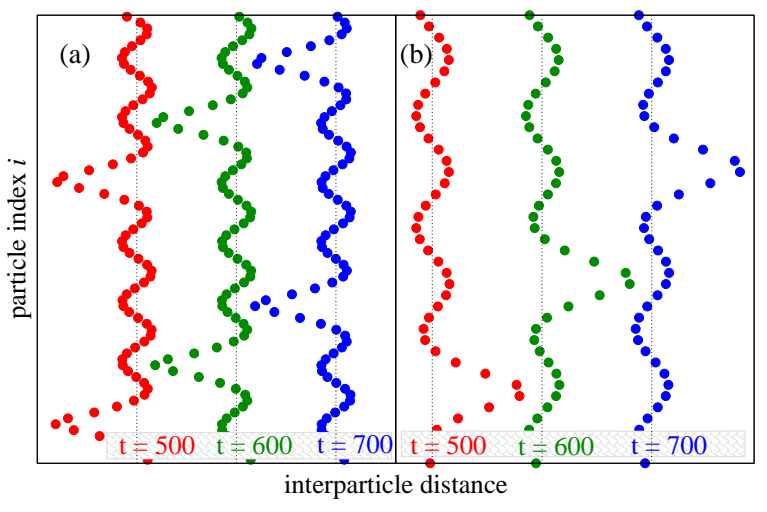

FIG. 2: (Color online) Snapshots of the distance between neighbor lubricant particles in the chain $x_{i}-x_{i-1}$ at three successive time frames. All parameters as in Fig. 1] except $r_{-} \simeq 10.36$, and $K=10$ (inside the main plateau). (a) $r_{+}=1.031\left(\right.$ kink density $\left.\delta / a_{+}=0.031\right) ;(\mathrm{b}) r_{+}=0.995$ (anti-kink density $\left.|\delta| / a_{+}=0.005\right)$.

We can understand these results as follows. Consider initially the situation of quasi-commensuration of the chain to the $a_{+}$substrate: $r_{+}=1+\delta$, with small $\delta$. This induces a density $\rho_{\text {sol }}=\delta / a_{+}$of solitons (or kinks, essentially substrate minima holding two particles, rather than one) [5]. The second, less oscillating $a_{-}$slider, which moves at velocity $v_{e x t}$, will $d r a g$ the kinks along: $v_{\text {sol }}=v_{\text {ext }}$. If $\rho_{0}=1 / a_{0}=r_{+} / a_{+}$ is the linear density of lubricant particles, mass transport will obey $v_{c m} \rho_{0}=v_{\text {sol }} \rho_{\text {sol }}$. This yields precisely $w=v_{c m} / v_{\text {ext }}=\rho_{\text {sol }} / \rho_{0}=\delta / r_{+}=1-r_{+}^{-1}$. Thus the exact plateaus arise because the smoother slider (whose exact periodicity $r_{-}>r_{+}$is irrelevant) drags the kinks, of given density, at its own full speed $v_{\text {ext }}$, as illustrated Fig. 27. As shown in Fig. 1 1 , this physics extends even to large $|\delta| \sim 1$, where no individual kink can be singled out. This works even for $\delta<0$ (anti-kinks), where, remarkably, the lubricant $\mathrm{CM}$ moves in the opposite direction with respect to the driving $v_{\text {ext }}(w<0$, see Fig. 1h for $\left.r_{+}=\phi^{-1}\right)$ : exactly as holes in a semiconductor, antikinks (carrying a negative "charge") moving at velocity $+v_{\text {ext }}$ effectively produce a backward net lubricant motion. The motion of the anti-kinks (regions of increased inter-particle separation) is illustrated in Fig. 2b.

The motion of particles is instructive. Figure 3 plots the time evolution of the velocity of a single chain particle $\dot{x}_{i}$, and of $v_{c m}$, for a value of $K$ inside a plateau, $K=1$, for $r_{+}=\phi$ and $r_{+}=\sigma$ of Fig. 1 Here two clear kinds of behavior emerge. Remarkably, the single-particle motion in the GM plateau is perfectly time-periodic. A similar periodic dynamics is found, in appropriate regimes, for all rational and quadratic irrational $\left(r_{+}, r_{-}\right)$values we tested. The SM motion is definitely not periodic. The Fourier spectrum of the particle motion, shown in Fig. [3; , confirm that the SM yields only quasi-periodic orbits with two prominent incommensurate frequencies 


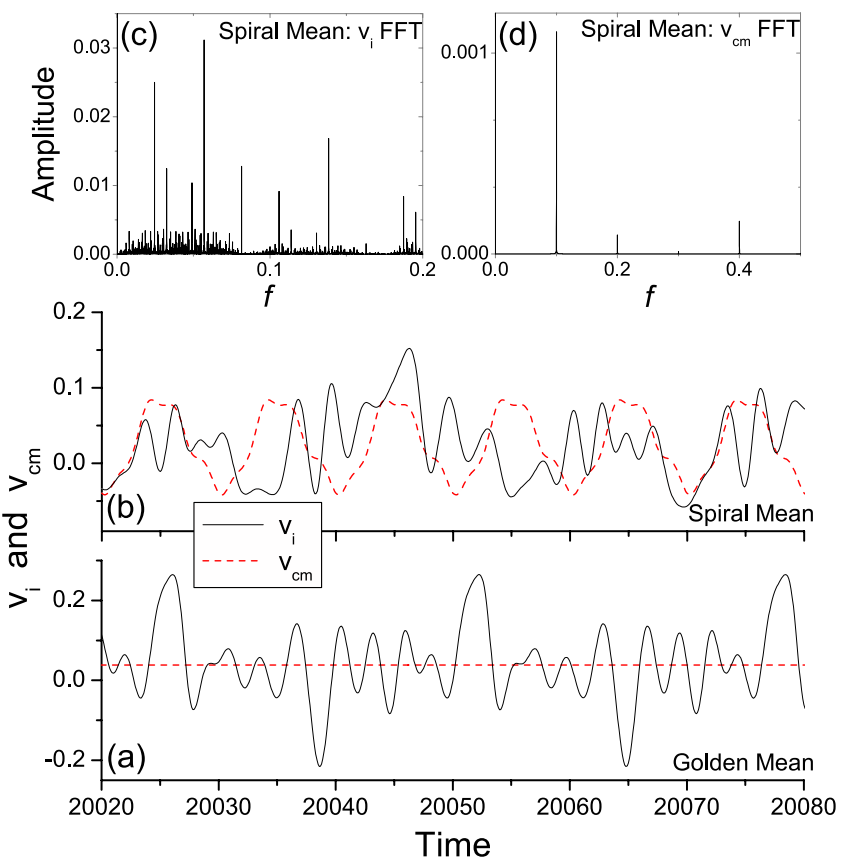

FIG. 3: (Color online) Time evolution of a particle velocity $\dot{x}_{i}$, and of the chain CM velocity $v_{c m}$ (fluctuations rescaled by a factor 50), for the GM (a) and SM (b) cases of Fig. 1] Amplitudes of the Fourier spectrum of $\dot{x}_{i}(t)$ (c) and of $v_{c m}(t)$ (d) for $r=\sigma$. Individual particle spectra have identical amplitudes, and differ only in the phases, which leads to a remarkable cancellation in the $v_{c m}$ power spectrum. Here $K=1, \gamma=0.1$, and $v_{\text {ext }}=0.1$.

$f_{+}$and $f_{-}$. However, a phase-cancellation between the Fourier spectra of different chain particles (all having the same amplitude spectrum, with different phases) yields strictly periodic CM motion. Periodic CM oscillations around an exactly quantized drift velocity is a common feature of all plateaus in the chain dynamics. These periodic oscillations can be understood as the solitons moving at velocity $v_{\text {ext }}$ encountering a periodic Peierls-Nabarro potential [5] of period $a_{+}$. The corresponding frequency of encounter $v_{\text {ext }} / a_{+}$is clearly visible in Fig. 31. We observe that the Peierls-Nabarro barrier vanishes (strictly constant $v_{c m}$ ) for all cases where the particle motion is periodic.

To understand why single particles may move periodically in some plateaus, let $f_{+}=v_{c m} / a_{+}$and $f_{-}=$ $\left(v_{e x t}-v_{c m}\right) / a_{-}$be the average encounter frequency of a generic particle with the two substrate periodic corrugations. Whenever these two frequencies are mutually commensurate,

$$
n_{+} f_{+}=n_{-} f_{-} \quad\left(\text { integers } n_{ \pm}\right),
$$

each particle is driven by the two generally incommensurate corrugations in an effectively periodic way, and undergoes a periodic oscillation, of period $T=n_{-} / f_{+}=$

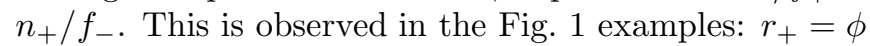
yields $n_{+} / n_{-}=1 / 1$ and $r_{+}=3 / 2$ yields $n_{+} / n_{-}=4 / 3$.

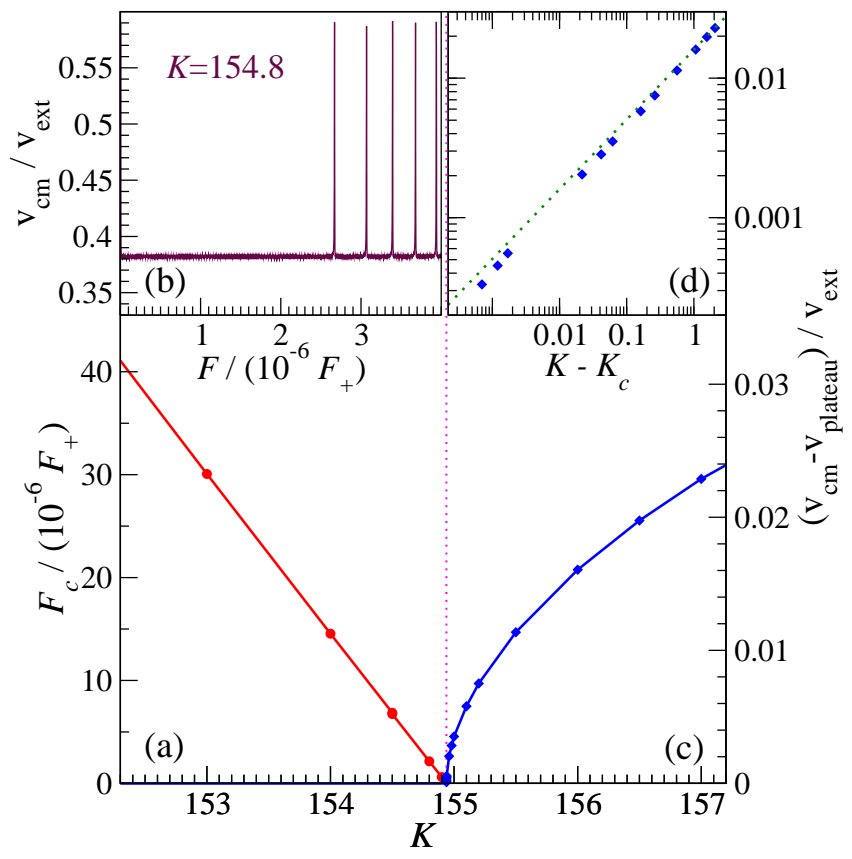

FIG. 4: (Color online) Dynamical depinning force $F_{c}$ for the GM plateau (a), extracted by a slow adiabatic increase of the force $F$ applied to all particles, until intermittencies appear, signaling collective slips (b). The critical behavior of the average velocity for $K \rightarrow K_{c}(\mathrm{c}, \mathrm{d})$, showing the typical square-root singularity (dotted line) associated to type-I intermittencies. Here $\gamma=0.1$, and $v_{e x t}=0.1$ were used.

The plateaus in Fig. 1 are labeled accordingly. When $f_{+}$and $f_{-}$are incommensurate, the motion is quasiperiodic, as illustrated by the SM case of Fig. 3 By definition, $f_{+} / f_{-}=\left(r_{-} / r_{+}\right) w /(1-w)$ : for any given plateau velocity $w$, suitable choices of $r_{-} / r_{+}$can make the two frequencies either commensurate, as in Eq. (2), or incommensurate. In particular, for the main plateau we have $f_{+} / f_{-}=r_{-}\left(r_{+}-1\right) / r_{+}$, which can be made rational for any choice of $r_{+}$, by choosing a suitable $r_{-}=r_{+}\left(r_{+}-1\right)^{-1} n_{-} / n_{+}$. For example, we verified that, for $r_{+}=\sigma$ and $r_{-}=\sigma(\sigma-1)^{-1}$, individual particles do oscillate periodically in the main plateau.

Low driving velocities $v_{e x t}$ are beneficial to the appearance and width of plateaus. For increasing $v_{e x t}$, the plateaus shrink and eventually disappear, still remaining exact while they do so. The critical $v_{e x t}$ where the plateaus end depends on $K$, but is usually smaller than unity, for the parameters of Fig. [1 The 1/1 plateau of the GM case is especially wide and robust against an increase of $v_{\text {ext }}$ (for the parameters of Fig. 1 and $K=4$, up to $v_{\text {ext }} \simeq 1.5$ ) and other perturbations. This seems related to $\phi$ being uniquely associated to the equal drivefrequency ratio, $n_{+} / n_{-}=1 / 1: \phi$ appears therefore, in the present dynamical context, as the "most commensurate" irrational, at variance with static pinning in the standard FK model, where the opposite is true [5, 7].

The finding of exact plateaus implies a kind of "dynam- 
ical incompressibility", namely identically null response to perturbations or fluctuations trying to deflect the CM velocity away from its quantized value. In order to probe the robustness of the plateau attractors, we introduced an additional constant force $F$ acting on all particles in the chain, trying to alter the force-free sliding in the plateau regime. As expected, as long as $F$ remains sufficiently small, it does perturb the single-particle motions but has no effect whatsoever on $w$, which remains exactly pinned to the attractor $\left(v_{c m} \equiv v_{\text {plateau }}\right)$. The plateau dynamics is only abandoned above a critical force $F_{c}$. This dynamical depinning takes place through a series of typeI intermittencies [8], as shown in Fig. 4b where $v_{c m}(t)$ is plotted against a slow adiabatic ramping of $F$.

A precise value of $F_{c}$ can be obtained by ramping $F$ with time with a gentle enough rate of increase or, alternatively, by a Floquet-Lyapunov linear stability analysis 9], to be shown elsewhere. The value of $F_{c}$ is a function of the parameters, and $F_{c}$ vanishes linearly when $K$ approaches the border $K_{c}$ of the plateau, as in Fig. 4 . The depinning transition line $F_{c}$, ending at $K=K_{c}$, is a 'first-order' line, with a jump $\Delta v$ in the average $v_{c m}$ and a clear hysteretic behavior (not shown) as $F$ crosses $F_{c}$. As can be expected, $\Delta v$ decreases to 0 as $K$ increases towards $K_{c}$. Thus $K=K_{c}$ represents a genuine non-equilibrium critical point, where the sliding chain enters or leaves a dynamical orbit. The precise value of $K_{c}$ depends on parameters such as $v_{\text {ext }}$ and $\gamma$; however, its properties do not. As $K$ approaches $K_{c}$ from above (no external force), $v_{c m}$ approaches $v_{\text {plateau }}$ in a critical manner, as suggested in Fig. 1] This is detailed in Fig. 4(c,d), where the critical behavior is shown to be $\Delta v \propto\left(K-K_{c}\right)^{1 / 2}$, the value typical of intermittencies of type I [8]. For $K \gtrsim K_{c}$, in fact the chain spends most of its time moving at $v_{c m}(t) \simeq v_{\text {plateau }}$, except for short bursts at regular time-intervals $\tau$, where the system as a whole jumps ahead by $a_{0}$, i.e. an extra chain lattice spacing (collective slip). The characteristic time $\tau$ between successive collective slips diverges as $\tau \propto\left(K-K_{c}\right)^{-1 / 2}$ for $K \rightarrow K_{c}$, consistent with the critical behavior of $w$. We verified that the $w$-plateaus for more general values of $r_{+}$and of $r_{-}$show the same kind of infinite stiffness, and a critical decrease of $F_{c}$ near the plateau edge, similar to that of Fig. 4 for the GM.

The phenomena just described for a model $1 \mathrm{D}$ system are quite extraordinary; it would be fascinating if they could be observed in real systems. Nested carbon nanotubes [10], or confined one-dimensional nanomechanical systems [11], are one possible arena for the phenomena described in this Letter. Though speculative at this stage, one obvious question is what aspects of the phenomenology just described might survive in twodimensions (2D), where tribological realizations, such as the sliding of two hard crystalline faces with, e.g., an interposed graphite flake, are conceivable. Our results suggests that the lattice of discommensurations - a
Moiré pattern- formed by the flake on a substrate, could be dragged by the other sliding crystal face, in such a manner that the speed of the flake as a whole would be smaller, and quantized. This would amount to the slider "ironing" the solitons onward. Dienwiebel et al. 12. demonstrated how incommensurability may lead to virtually friction-free sliding in such a case, but no measure was obtained for the flake relative sliding velocity. Real substrates are, unlike our model, not rigid, subject to thermal expansion, etc. Nevertheless the ubiquity of plateaus shown in Fig. 11 and their topological origin, suggests that these effects would not remove the phenomenon. A real-life situation with a distribution of differently oriented crystalline micro-grains, each possessing a different incommensurability, is also potentially interesting; each grain, we expect, will tend to stabilize a certain average CM velocity depending on its incommensurability. Other realizations or applications inspired by the physics described by our model might be accessible, notably in grain boundary motion, in the sliding of optical lattices [13] or of charge-density-wave systems [14].

Acknowledgments - We are grateful to O.M. Braun for invaluable discussions. This research was partially supported by PRRIITT (Regione Emilia Romagna), NetLab "Surfaces \& Coatings for Advanced Mechanics and Nanomechanics" (SUP\&RMAN) and by MIUR Cofin 2004023199, FIRB RBAU017S8R, and RBAU01LX5H.

[1] A.E. Filippov, J. Klafter, and M. Urbakh, Phys. Rev. Lett. 87, 275506 (2001).

[2] T. S. van Erp, A. Fasolino, O. Radulescu, and T. Janssen, Phys. Rev. B 60, 6522 (1999).

[3] A. Vanossi, J. Röder, A.R. Bishop, and V. Bortolani, Phys. Rev. E 63, 017203 (2000).

[4] PBC are realized either by (i) approximating $a_{-} / a_{+}$and $r_{+}=a_{+} / a_{0}$ with suitable rational numbers $N_{+} / N_{-}$and $N / N_{+}$, or (ii) by using a finite $N$ and machine-precision values of $r_{+}$and $r_{-}$. Both methods introduce a finite-size error, which can be made arbitrarily small by choosing suitably large integers $N, N_{ \pm}$, and lead to the same results for large $N, N_{ \pm}$, where full incommensurability is restored.

[5] O. M. Braun and Yu. S. Kivshar, The Frenkel-Kontorova Model: Concepts, Methods, and Applications, SpringerVerlag, Berlin (2004).

[6] O. M. Braun, A. Vanossi, and E. Tosatti, Phys. Rev. Lett. 95, 026102 (2005).

[7] M. Peyrard and S. Aubry, J. Phys. C: Solid State Phys. 16, 1593 (1983).

[8] P. Bergé, Y. Pomeau, and C. Vidal, Order within Chaos (Hermani and John Wiley \& Sons, Paris, 1984).

[9] J. V. José and E. J. Saletan, Classical dynamics: a contemporary approach (Cambridge University Press, Cambridge, 1998).

[10] X.H. Zhang, U. Tartaglino, E. Tosatti (unpublished).

[11] B. Toudic, et al., Phys. Rev. Lett. 96, 145503 (2006).

[12] M. Dienwiebel, et al., Phys. Rev. Lett. 92, 126101 (2004). 
[13] I. Bloch, Nature Physics 1, 23 (2005).

[14] G. Grüner, Rev. Mod. Phys. 60, 1129 (1988). 